\title{
Power inverter design for ASDEX Upgrade saddle coils
}

\author{
M.Teschke*, W. Suttrop, M. Rott and the ASDEX Upgrade Team \\ Max Planck Institute for Plasma Physics, \\ EURATOM Association, Boltzmannstr. 2, 85748 Garching, Germany
}

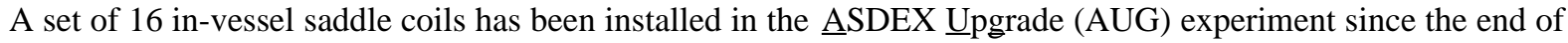
2011 [1]. To achieve full performance, it is necessary to operate them with alternating current (AC) of arbitrary waveforms. To generate spatially resolved magnetic fields, it is required to allocate separate power inverters to every single coil. Therefore, different topologies are analyzed and compared. Studies of the commutation behaviour of power stages, different pulse width modulation (PWM) schemes and single-phase-to-earth fault detection are executed. Experiments to evaluate the electromagnetic interference (EMI) of possible inverter topologies on the AUG diagnostics are done as well. A special focus is put on the feasibility of analyzed topologies using industrially available and fully assembled "power modules" to minimize development effort and costs.
\end{abstract}

Keywords: RWM; ELM; Power Inverter; Power Block; NPC; FC; Cascade; PWM; CM current; EMI

\section{Introduction}

Two different sets of magnetic perturbation coils are planned or already integrated into AUG's tokamak - the so called "B-Coils" and the "A-coils" [1]. Currently, only the B-coils are integrated and driven by direct current (DC) for first experiments. The aim is to mitigate different kind of plasma instabilities - the "edge localized modes" (ELM) [2] and the resistive wall modes (RWM) [3] using an active feedback control mechanism [4]. To get the full performance, it is necessary to operate each coil independently with arbitrary AC waveforms. Therefore, up to 24 power inverters are needed. Although, it is planned to realize the B-coil inverters first, it is advantageous in terms of development time and costs to find an inverter concept that can easily be adapted to the A-coils. The peak value of current has to be limited to $1 \mathrm{kA}$ due to IxB forces. The bandwidth of the B-coils is $500 \mathrm{~Hz}$ and the bandwidth of the A-coils will be in the range of $3 \mathrm{kHz}$. The minimal operation voltage results from coil impedance. For the B-coils it is in the range of $100 \mathrm{~V}$. A higher voltage has a higher current ripple as a consequence.

\section{Choice of inverter topology}

A modular concept for AUG consisting of several, identical "power blocks" has been developed previously $[5,6]$. In this proposal, two power blocks are needed to drive an A-coil and only one to drive a B-coil with limited bandwidth (see Fig. 1). To operate A-coils, the so called "inverter cascade" is used [7]. An important advantage of this concept is multi-voltage-level operation. A single power block generates three voltage levels, only $\left(+\mathrm{V}_{\mathrm{DC}},-\mathrm{V}_{\mathrm{DC}}\right.$ and $\left.0 \mathrm{~V}\right)$. The inverter cascade is able to generate five levels $\left(-2 \mathrm{~V}_{\mathrm{DC}},-1 \mathrm{~V}_{\mathrm{DC}}, 0 \mathrm{~V},+1 \mathrm{~V}_{\mathrm{DC}}\right.$, $+2 V_{D C}$ ). Furthermore, the effective switching frequency can be doubled by phase shifted operation of the power blocks. This is possible because a given output voltage can be achieved by different, redundant switching states of the power blocks (16 switching states for five voltage levels). In case of B-coils, the bandwidth, the required current gradients and the operating voltage are smaller. Thus, a single power block operation had been proposed for them.

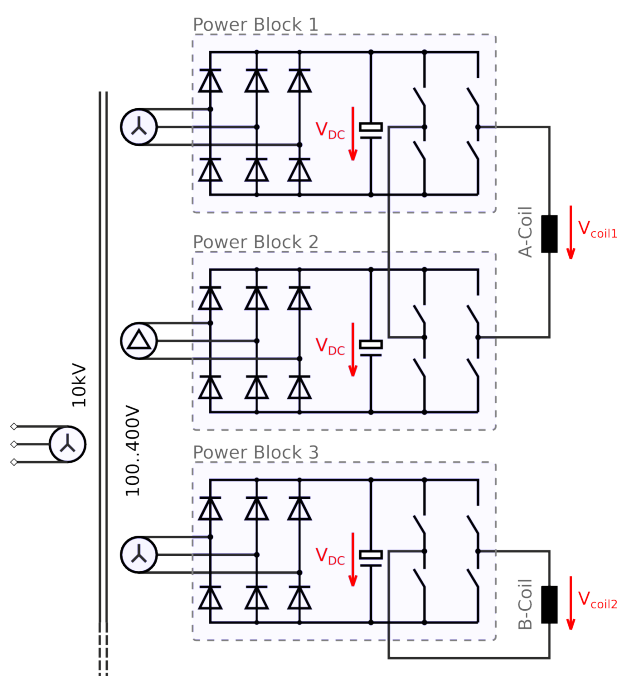

Fig. 1 Inverter cascade and 3-level full bridge to operate A- and B-coils.

Apart from these advantages, there are some limitations. The DC links of the power blocks have to be floating voltages. Therefore, every power block is connected by a separate transformer to the power grid. Alternatively, big transformers with several isolated secondary windings can feed several power blocks. Both are expensive and sharing of reactive power stored in different DC links becomes difficult. To do this, the input rectifier of any power block has to be replaced by a more complex four quadrant type to allow positive and negative currents. However, sharing of reactive power is economically very advantageous for this application, because hundreds of kVA per coil have to be exchanged permanently between coil and its inverter. These requirements lead to high costs.

To drive the B-coils it is not necessary to decouple the DC links of the full bridges. They can directly be powered by H-bridges coupled to a powerful, common DC link. This is shown in Fig. 2. Reactive power can be 
exchanged via this link. Unfortunately this is only a solution for the B-coils. It is impossible to extend this concept to multi-level operation or to increase the frequency by phase shift switching method for the Acoils. Furthermore, experiments presented in chapter 5 show that multi-level operation can be useful for B-coils, too. But there is another disadvantage of the H-bridge operation that can not be tolerated for either coil type: It has to be possible to set both inverter output joints to ground potential. This is not possible for the H-bridge without strong disadvantages, as described in chapter 4 .

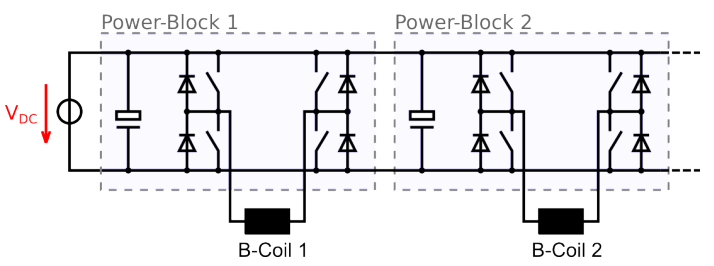

Fig. 2 H-Bridge operation of B-coils.

In Fig. 3, two alternative topologies for multi level inverters are presented - the neutral point clamped (NPC) and the flying capacitor (FC) topology. Both concepts are suited for galvanic coupling of several DC links to a single powerful DC link. Both are commonly adopted in a wide range of applications [8]. Related to development costs, it is a big advantage if these concepts are realizable by use of commonly available power electronic modules. This approach leads to development of the topology presented in Fig. 4. In case of removing the green dotted DC link capacities of power blocks 3 and 6 and leaving the other blocks in their original state (blue dotted connections not removed), the arrangement can be used as NPC topology. The switches $\mathrm{S} 1_{\mathrm{L}}, \mathrm{S} 2_{\mathrm{U}}$, $\mathrm{S} 4_{\mathrm{L}}$ and $\mathrm{S} 5_{\mathrm{U}}$ are deactivated but their free wheeling diodes are needed to connect the middle potential of the common DC link to the load. a)

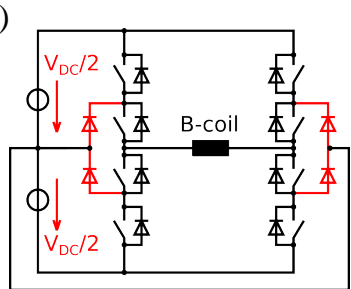

b)

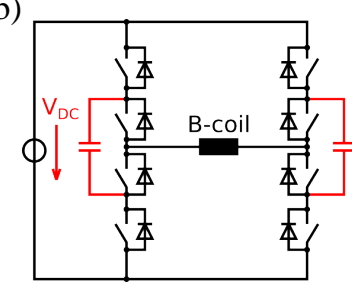

Fig. 3 (a) NPC- and (b) FC-multi level inverter topologies.

The FC topology is realizable by disconnecting $\mathrm{S} 1_{\mathrm{L}}$, $\mathrm{S} 2_{\mathrm{U}}, \mathrm{S} 4_{\mathrm{L}}$ and $\mathrm{S} 5_{\mathrm{U}}$ from their DC links (blue dotted connections removed). The green dotted DC link capacities of power blocks 3 and 6 remain activated.

This approach seems to be an elegant way to realize a complex topology by the help of commercially available and fully developed power modules. But its realization is not without risk. The electric connections between power block 1 and 2 with the "flying" power block 3 conduct highly variable currents. Stray inductance plays an important role. It has to be minimized as much as possible. The same is true for the electric connections between power blocks 4 and 5 to their flying power block 6.

In case of NPC topology, the DC link voltages of the flying power blocks 3 and 6 are highly variable. Proper operation of commercial power blocks is not warranted in this case. Thus, we will perform simple experiments with a minimal number of power blocks to demonstrate feasibility.

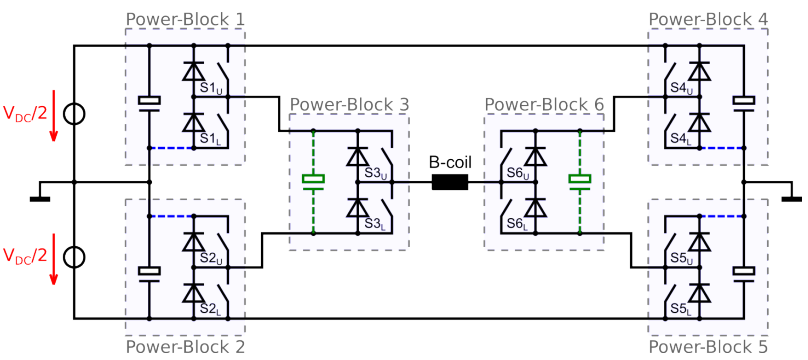

Fig. 4 NPC and FC topologies realized with commonly available power blocks.

\section{PWM scheme}

Especially for NPC topology realized with power blocks (Fig. 4), the PWM scheme was analyzed in detail and compared with the originally proposed inverter cascade (Fig. 1, upper two power blocks). If the switches $\mathrm{S} 1_{\mathrm{L}}, \mathrm{S} 2_{\mathrm{U}}, \mathrm{S} 4_{\mathrm{L}}$ and $\mathrm{S} 5_{\mathrm{U}}$ remain activated and the common DC link is stabilized by two voltage sources $\mathrm{V}_{\mathrm{DC}} / 2$ (called "advanced NPC" in the following), the switching behaves very similar to the inverter cascade. There also exist 16 switching states to generate five voltage levels at the output. This is shown in Table 1. "P" describes a switching state of a power block with upper switch on (e.g. $\mathrm{S} 1_{\mathrm{U}}$ on and $\mathrm{S} 1_{\mathrm{L}}$ off) and "N" with lower switch on (e.g. $S 1_{U}$ off and $S 1_{L}$ on). For a five level advanced NPC full bridge, there exists one switching state for a load voltage of $+V_{D C}$, four switching states for $+V_{D C} / 2$, six for zero output, four for $-\mathrm{V}_{\mathrm{DC}} / 2$ and one for $-\mathrm{V}_{\mathrm{DC}}$. This is exactly the same for the inverter cascade. As a result, a well chosen PWM scheme can be used to double effective switching frequency. This is very interesting four the given application because of the high bandwidth of A-coils. The scheme is shown as an example in the right column of Table 1. PWM is typically done between neighboring voltage vectors to realize a load voltage level between the two voltage vectors in average, e.g. between $+\mathrm{V}_{\mathrm{DC}} / 2$ and $+\mathrm{V}_{\mathrm{DC}}$ (="Group 1"). The switching scheme for each group demonstrates that every switch toggles only once for four variations in output voltage. This means that the switching frequency of inverters output can be up to four times higher than that for a single power block.

Another question is the commutation behaviour of load current. A detailed analysis comes to the result that half of the commutation processes can be performed by zero voltage switching (ZVS), called "natural commutation" as well. This is the best possible situation for non-resonant power inverters and it is the same value as for the inverter cascade.

As an example, this is shown for switching Group 2 in Fig. 5. Red marked half bridges perform hard switching. This means, the neighboring switch has to commutate load current at full voltage. During commutation process, a lossy short circuit on a conducting free wheel diode occurs. Yellow marked half bridges perform the natural commutation. This means, commutation was initiated by an interplay of free wheeling diodes and finally the power switch takes the load current at a very small voltage level (= flux voltage 
of a diode). In this case, commutation losses have a minimum.

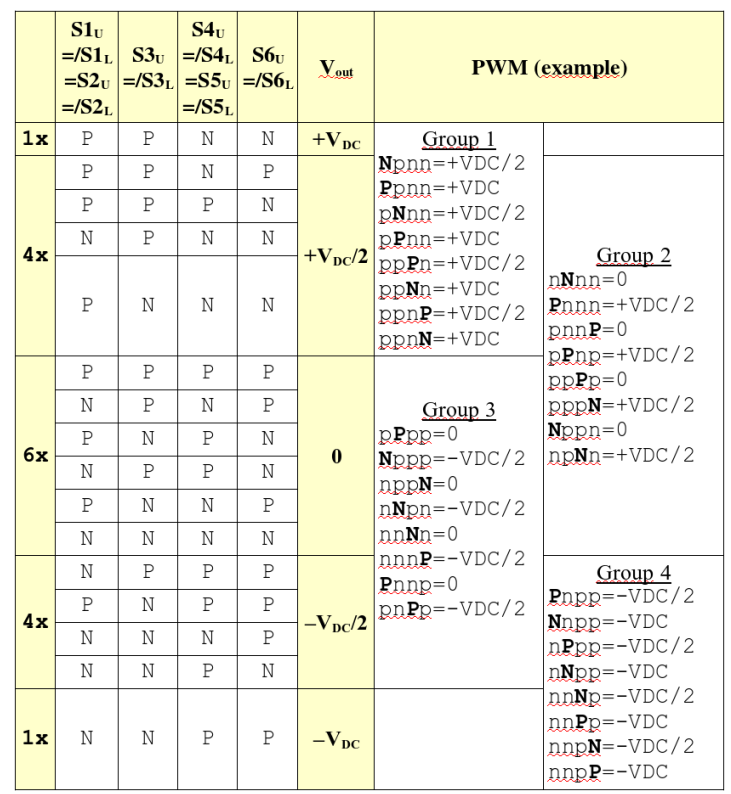

Table 1 Switching states and PWM scheme for the advanced NPC topology.

\section{Protection of the gas isolated feed-through}

The coils are an integrated part of the ultra high vacuum torus-shaped vessel of the AUG experiment. The electric connections are fed through $1.3 \mathrm{~m}$ long tubes pumped down to an intermediate pressure in the range of $10^{-4} \ldots 10^{-2}$ mbar. Experiments have shown that the breakdown voltage of the feed-through significantly depends on the magnetic field strength that can reach a value of up to 3 Tesla. The breakdown voltage without presence of magnetic fields is in the range of $2 . .3 \mathrm{kV}$. During the experiment, it decreases to a value of several $100 \mathrm{~V}$, as shown in the PASCHEN-like curve of Fig. 6. Here, every coloured dot stands for a breakdown experiment performed during a plasma shot.

An electric breakdown resulting in a strong electric arc can have hard consequences. In the worst case, a vacuum leakage into the main chamber occurs and the whole experiment has to be stopped for several weeks.

An important result of these breakdown experiments is that electrical isolation is good enough during normal operation. But the safety factor is much smaller than for other, typical high voltage applications. Thus, a good detection of fault-operation in combination with an effective strategy for fast turn-off becomes very important.

The occurrence of an arc in the feed-through always results in high currents conducted by the main vessel housing back to the voltage source (= inverter's DC link). This common mode (CM) current can be detected if a single connection between DC link voltage and vessel potential exists. Then, a good response to the event is not to ramp down the current as fast as possible. Eliminating the arc's voltage to immediately limit its energy consumption is more efficient. Of course, load current continues to circulate through free wheeling diodes and switches. They have to be able to tolerate this.

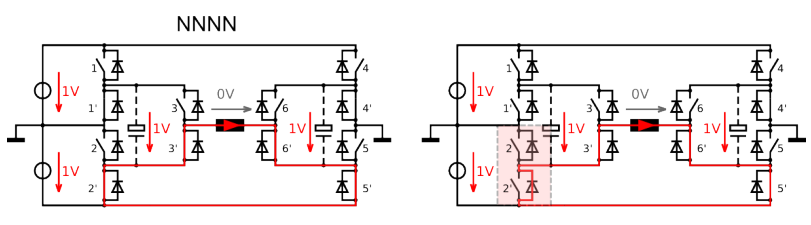

PNNN

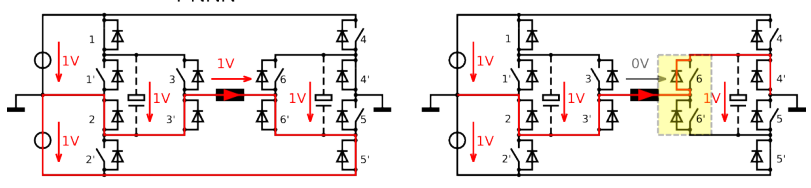

PNNP

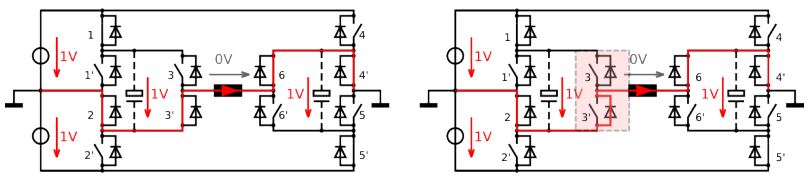

PPNP

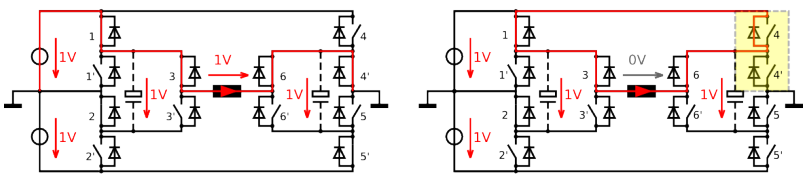

PPPP

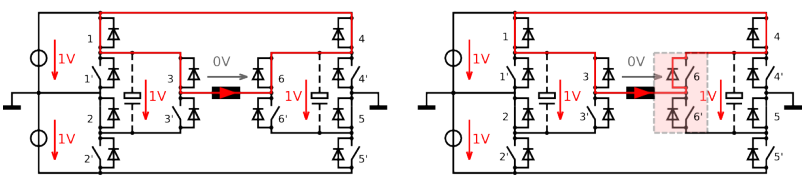

PPPN

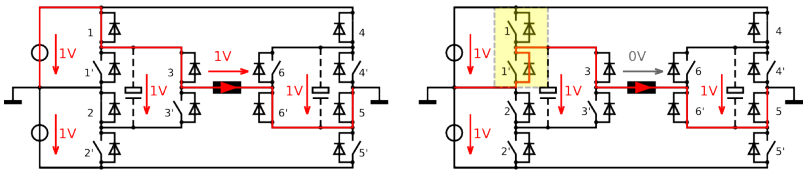

NPPN

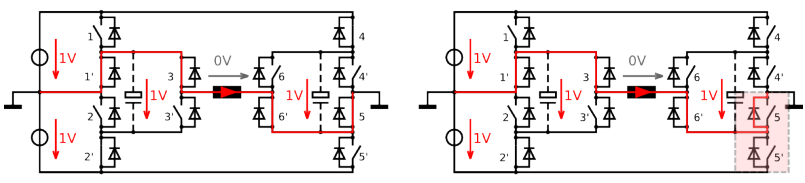

NPNN

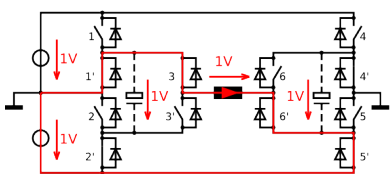

Fig. 5 Commutation behaviour in switching Group 2 of the presented PWM scheme in table 1.

This method can only be applied, if an inverter topology is chosen where both load connections are switchable to the vessel potential. For the NPC topology (Fig. 4) this is e.g. possible by turning on $\mathrm{S} 1_{\mathrm{L}}, \mathrm{S} 3_{\mathrm{U}}, \mathrm{S} 6_{\mathrm{U}}$ and $S 4_{L}$. For the H-bridge topology (Fig. 2) this is only possible, if the positive or negative terminal of DC link is connected to vessel potential. Unfortunately, this leads to an asymmetric electric situation and an increase of EMI to the whole electric environment would be an unpleasant consequence.

The realization of a fast $\mathrm{CM}$ current detection is not trivial because CM currents are continuously generated at normal operation by capacitively conducted currents during switching events. These events have to be blanked in a fail-safe manner. Corresponding experiments have currently started. 


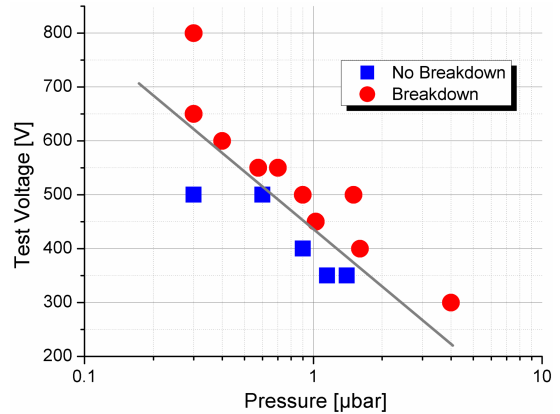

Fig. 6 Breakdown voltage of feed through during plasma shots with high magnetic field strength between $2.4 \ldots 2.5 \mathrm{~T}$.

\section{Electromagnetic (EM) noise measurements}

Before realization of a complex inverter system, its expected influence to AUG's manifold and highly sensitive diagnostics was analyzed. Therefore, the output of a low power switched mode power inverter was connected single-sided to each B-coil as shown in Fig. 7. By use of a variable ratio transformer, its DC link voltage and thus, its output peak voltage is variable between 50 and $500 \mathrm{~V}$. This imitates the different EM behaviour of inverter topologies with a different number of voltage levels on stray capacities. Inverter's switching frequency is selectable between 5 and $10 \mathrm{kHz}$. The sum of all stray capacities is in the range of $1 \mu \mathrm{F}$ and the RMS-value of CM current can be up to $100 \mathrm{~A}$, depending on output voltage level and velocity of switching events. Capacitively coupled CM currents to the vessel housing should be the main source for EM noise, because switching events are not too short (> 1 $\mu$ s) and thus the bandwidth is limited to several $100 \mathrm{kHz}$. Thus, EM radiation becomes very inefficient.

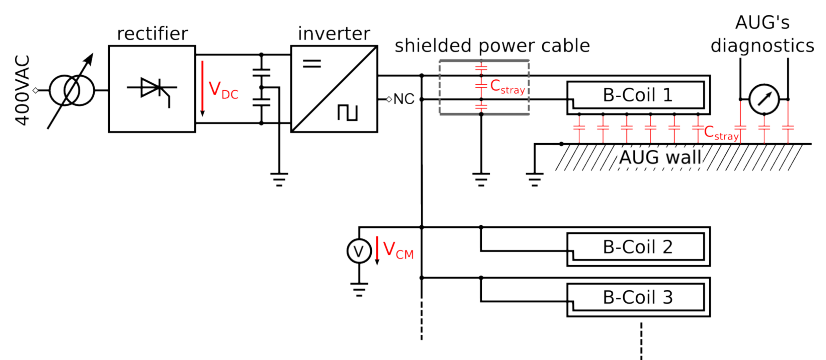

Fig. 7 EMI test circuitry.

As a first result of the experiment, seven different kind of diagnostic groups received intense noise clearly transmitted by the B-coils. The signal to noise ratio (SNR) of the most sensitive diagnostic (diode bolometry) reached a value of 0.05 . This means, noise amplitude was up to 20 times higher than the signal (see Fig. 8)! After several variations of parameters, representing different inverter topologies with e.g. different rate of voltage step at the output, two critical diagnostics with an SNR value down to 1.0 remained. Here, electric modification of the diagnostic itself will be unavoidable.

An important outcome of these experiments is, that multi-level operation can be advantageous for A- and Bcoils. The more voltage levels an inverter generates, the smaller is the voltage gradient during PWM operation and the smaller are the induced capacitive stray currents $\left(\mathrm{i}_{\mathrm{C}}=\mathrm{C} \cdot \mathrm{du}_{\mathrm{C}} / \mathrm{dt}\right)$.
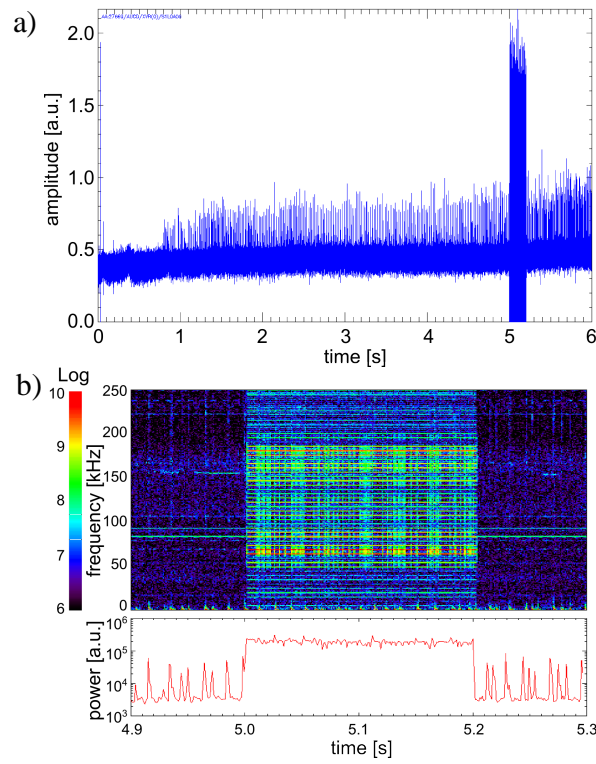

Fig 8 (a) Time trace of bolometry XVR-S1L0A00 during plasma shot \#27.669. The EMI test was activated at 5...5.2 sec.

(b) Spectrogram of (a). EM noise is generated with a bandwidth of several hundred $\mathrm{kHz}$.

\section{Conclusions and Outlook}

An appropriate inverter topology to drive the recently installed magnetic perturbation coils of AUG has been found. Control and balancing of losses is possible by choice of good PWM schemes in case of the "advanced NPC" topology, we currently started to realize. Exchange of reactive power between multiple inverters is possible as well. A big advantage is the possibility to use commercially available power modules. The influence of switched mode power supplies on AUG's highly-sensitive diagnostics was analyzed by several experiments, too. Some of them require multi-level inverter topologies to increase SNR value to an acceptable level. Another important question is the peak amplitude of operating voltage of the coils. It was found, that its insulation voltage significantly depends on applied magnetic field. Thus, a fast detection of fault operation and a good strategy for fast turn-off is needed. The next goal is to perform first experiments by use of the proposed inverter topologies. Therefore, a small number of power blocks were purchased. A PCbased approach for the fast current controller and PWM unit is aimed. Specialized interface cards have to be developed to connect the PC hardware with the power blocks and to provide safety features.

\section{References}

[1] M. Rott et al., Fus. Eng. Des. 84 (2009), pp. 1653-1657

[2] W. Suttrop et al., Plas. Phys. Contr. Fus. 53 (2011), DOI 124014

[3] Strumberger et al., Phys. Plas. 15 (2008), DOI 2884579

[4] W. Suttrop et al., EPS Conf.Plas.Phys.33E (2009), pp. 1165

[5] E. Gaio et al., Fus.Eng.Des. 86 (2011), pp. 1488-1492

[6] A. Zamengo, Diss.Univ.Padova/Italy,Dep.Elec.Eng. (2011), http://paduaresearch.cab.unipd.it/3516/1/PhD_azamengo.pdf

[7] Malinowski et al., IEEE Trans. Ind. Elec. 57 (2010), pp. 2197-2206

[8] S. Fazel et al., IEEE Trans.Ind.App.43(2007),pp.1032-1040 